\title{
Brain Tumour Detection and Classification using Fusion Technology on IOT
}

\author{
K. Malarvizhi ${ }^{1}$, P.Ramya Devi ${ }^{2}$, Akshay G Bhat ${ }^{3}$ \\ Associate Professor, Department of CSE, JCT College of Engineering and Technology, Coimbatore \\ U.G. Student, Department of CSE, JCT College of Engineering and Technology, Coimbatore ${ }^{2,3}$
}

\begin{abstract}
Automatic defects detection in MR images is very important in many diagnostic and therapeutic applications. Because of high quantity data in MR images and blurred boundaries, tumour segmentation and classification is very hard. This work has introduced one automatic brain tumour detection method to increase the accuracy and yield and decrease the diagnosis time. The goal is classifying the tissues to three classes of normal, begin and malignant. In MR images, the amount of data is too much for manual interpretation and analysis. During past few years, brain tumour segmentation in Magnetic Resonance Imaging (MRI) has become an emergent research area in the field of medical imaging system. In the proposed system accurate detection of size and location of brain tumour plays a vital role in the diagnosis of tumour. The diagnosis method consists of four stages, pre-processing of MR images, feature extraction, and classification. The features selection is based on Discrete Wavelet Transformation (DWT).and feature extraction based GLCM. In the last stage, Probabilistic Neural network is employed to classify the Normal and abnormal brain. After that normal image store on cloud and move towards on android application.
\end{abstract}

Keywords: Brain Tumour detection, MRI Scan, DVM, GLCM, PNN, Deep learning, cloud access, feature selection, feature selection

\section{INTRODUCTION}

The brain is the management centre of the central nervous system and is responsible for the execution of activities all throughout the human body. Brain tumours can threaten human life directly. If the tumour is detected at an early stage, the patient's survival chance increases. Magnetic resonance (MR) imaging is widely used by physicians in order to determine the existence of tumours or the specification of the tumours [1]. The qualification of the brain cancer treatment depends on the physician's experience and knowledge [2]. For this reason, using an automated and flawless working tumour detection system is extremely important to aid physicians to detect brain tumours.

Detection of tumours in the brain via MR images has become an important task and numerous studies have been conducted in recent years. The flaws in MRI scan images could result in wrong scan results and this can't happen as it's a matter of life. So in here, we are considering filtering the results of an MRI scan using three discrete methods namely, Discrete Wavelength Transformation (DWT), Grey Level Co-Occurrence Matrix (GLCM), and Probabilistic Neural Network (PNN). After matching the search results with those of the MRI images stored in the database for reference, an accurate result will be returned. This result is further allowed to be accessed by the patient/a doctor by cloud access and from there to a discrete android mobile application.

The existing system for Brain Tumor Detection mostly consists of methods such as threshold techniques and edge detection. Image processing is any form, of information processing, in which the input is an image. The existing method is based on the threshold and also region growing. At the threshold based segmentation the image is considered as having only two values either black or white. But the bitmap image contains 0 to 255 gray scale values. So it ignores the tumor cells also. In [1] case of the region growing based segmentation it needs more user interaction for the selection of the seed. Seed is nothing but the center of the tumor cells; it may cause intensity in homogeneity problems. And also it will not provide the acceptable result for all the images.

The regional growing method ignored the spatial characteristics. Normally spatial characteristics are important for malignant tumor detection. In [4] threshold based segmentation the image is considered as having only two values either black or white. This is the main problem of the current system, due to that proposed technique for brain tumor segmentation. Hence, due to the lack of filtering accuracy, the scan results may go faulty, resulting in a wrong result. These methods are not applicable for multiple images for tumor detection in a short time. And also, Medical Resonance images contain a noise caused by operator performance which can lead to serious in accuracies classification. 


\section{SYSTEM DESIGN}

Usage of filtering and image processing methods such as Discrete Wavelength Transform (DWT), Grey Level CoOccurrence Matrix (GLCM), and Probabilistic Neural Network (PNN) are used for image filtration and tumour detection. The MRI scan image is subjected for a pre-processing and then is filtered using discrete wavelength transform algorithm. The processed result of DWT is fed as input to the GLCM method. The GLCM features are given input to the PNN classifier along with a matching algorithm to match the sample images stored in the database by future extraction. By comparing these results, we can arrive at a conclusion of whether the given image is of an affected person or not.

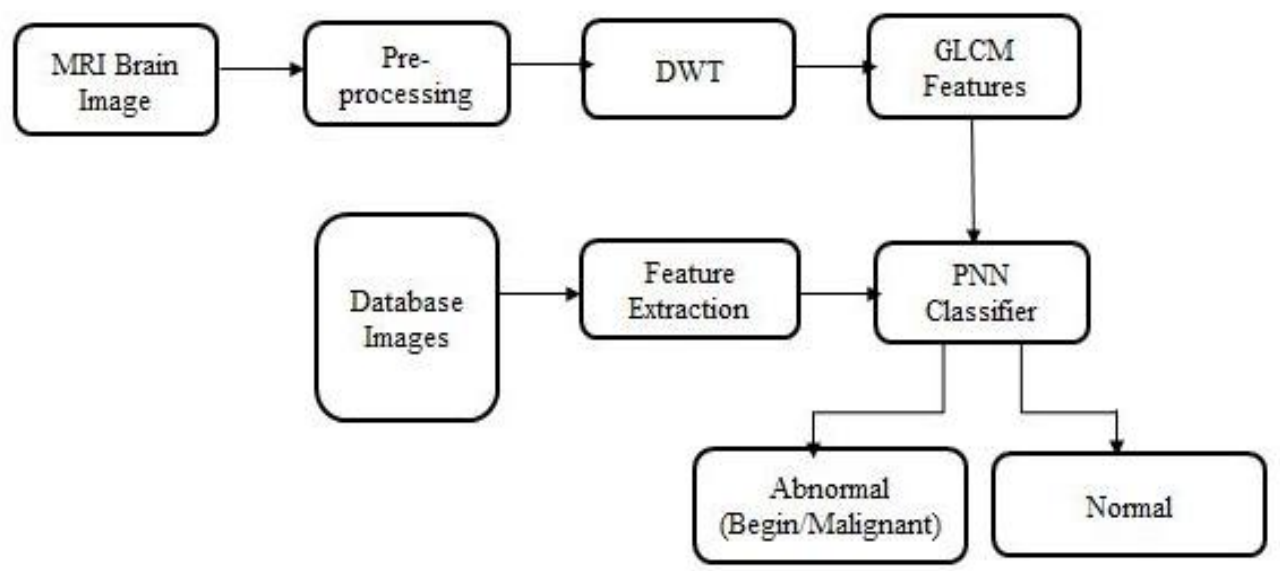

Figure 1 Brain Tumour Detection

\section{BRAIN TUMOUR DETECTION AND CLASSIFICATION}

Automatic brain tumour detection is to increase the accuracy and yield and decrease the diagnosis time. The goal is classifying the tissues to three classes of normal, begin and malignant. In MR images, the amount of data is too much for manual interpretation and analysis. During past few years, brain tumour segmentation in magnetic resonance imaging (MRI) has become an emergent research area in the field of medical imaging system. In the proposed system accurate detection of size and location of brain tumour plays a vital role in the diagnosis of tumour. The diagnosis method consists of four stages, pre-processing of MR images, feature extraction, and classification. The features selection is based on discrete wavelet transformation (DWT).and feature extraction based GLCM. In the last stage, Probabilistic Neural network is employed to classify the Normal and abnormal brain. After that normal image store on cloud and move towards on android application

\section{A. Discrete Wavelength Transform (DWT)}

The dual-tree complex DWT of a signal $\mathrm{x}$ is implemented using two critically-sampled DWTs in parallel on the same data. The transform is 2-timesexpansive because for an N-point signal it gives 2NDWT coefficients. If the filters in the upper and lower DWTs are the same, then no advantage is gained. However, if the filters are designed is a specific way, then the sub band signals of the upper DWT can be interpreted as the real part of a complex wavelet transform, and sub band signals of the lower DWT can be interpreted as the imaginary part. Equivalently, for specially designed sets of filters, the wavelet associated with the upper DWT can be an approximate Hilbert transform of the wavelet associated with the lower DWT. When designed in this way, the dual-tree complex DWT is nearly shift-invariant, in contrast with the critically-sampled DWT.

Moreover, the dual-tree complex DWT can be used to implement 2D wavelet transforms where each wavelet is oriented, which is especially useful for image processing. (For the separable 2D DWT, recall that one of the three wavelets does not have a dominant orientation.) The dual-tree complex DWT outperforms the critically-sampled DWT for applications like image de-noising and enhancement.As with other wavelet transforms, a key advantage it has over Fourier transforms is temporal resolution it captures both frequency and location information (location in time).

The Haar wavelet transform may be considered to pair up input values, storing the difference and passing the sum.This process is repeated recursively, pairing up the sums to prove the next scale, which leads to differences and a final sum. 


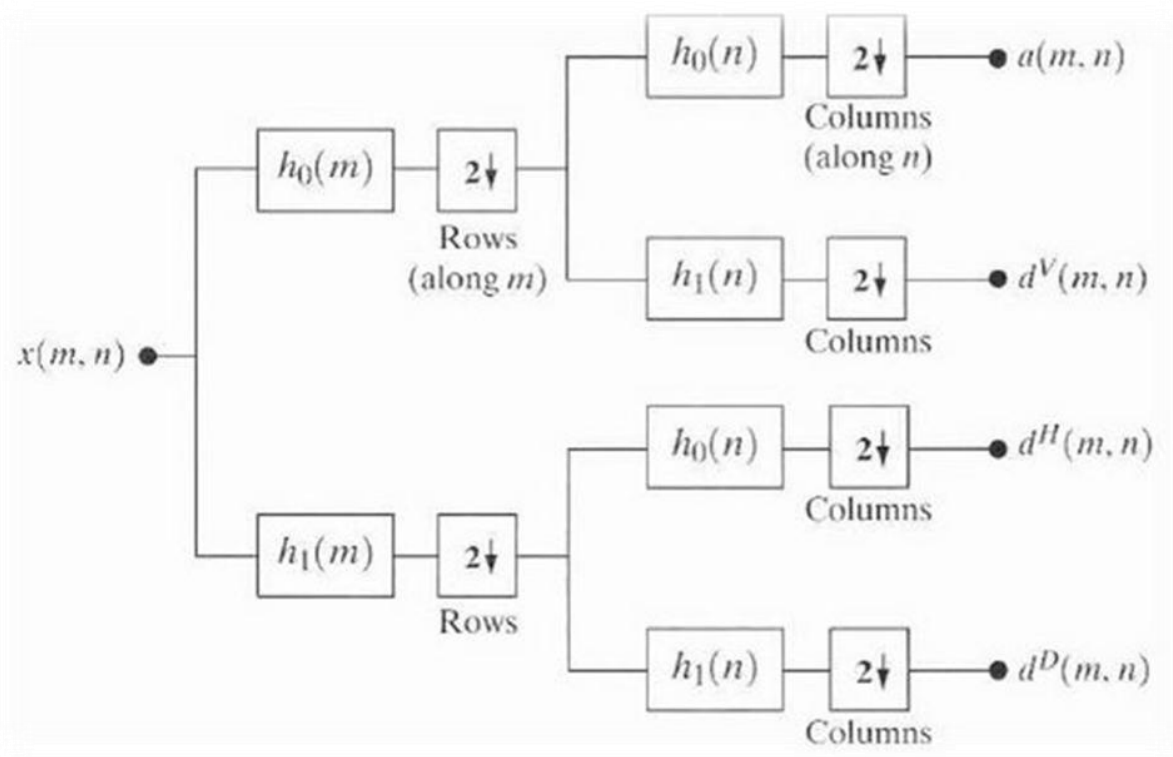

Figure 2 Discrete Wave length Transform

\section{B. Grey Level Co-Occurance Matrix (GLCM) Method}

The GLCM method is also referred as co-occurrencedistribution.It is the most classical second-order statistical method for texture analysis. An image is composed of pixels each with an intensity (a specific gray level), the GLCM is a tabulation of how often different combinations of gray levels co-occur in an image or image section.Texture feature calculations use the contents of the GLCM to give a measure of the variation in intensity at the pixel of interest. GLCM texture feature operator produces a virtual variable which represents a specified texture calculation on a single beam echogram.

\section{Steps for virtual variable creation:}

- Quantize the image data: Each sample on the echogram is treated as a single image pixel and its value is the intensity of that pixel. These intensities are then further quantized into a specified number of discrete gray levels, known as Quantization.

- Create the GLCM: It will be a square matrix $\mathrm{N} \mathrm{x} \mathrm{N}$ in size where $\mathrm{N}$ is the Number of levels specified under Quantization.

- $\quad$ Steps for matrix creation are:

- Let $\mathrm{s}$ be the sample under consideration for the calculation.

- Let $\mathrm{W}$ be the set of samples surrounding sample $\mathrm{s}$ which fall within a window centered upon sample $\mathrm{s}$ of the size specified under Window Size.

- Define each element i, $j$ of the GLCM of sample present in set $W$, as the number of times two samples of intensities $i$ and $j$ occur in specified Spatial relationship.

- The sum of all the elements $i, j$ of the GLCM will be the total number of times the specified spatial relationship occurs in $\mathrm{W}$.

- Make the GLCM symmetric: Make a transposed copy of the GLCM and add this copy to the GLCM itself.

- This produces a symmetric matrix in which the relationship $i$ to $j$ is indistinguishable for the relationship $j$ to i.Due to summation of all the elements $i, j$ of the GLCM will now be twice the total number of times the specified spatial relationship occurs in $\mathrm{W}$.

- Normalize the GLCM: Divide each element by the sum of all elements. The elements of the GLCM may now be considered probabilities of finding the relationship $i, j($ or $j, i)$ in $\mathrm{W}$.

- Define each element i, $j$ of the GLCM of sample present in set $W$, as the number of times two samples of intensities $\mathrm{i}$ and $\mathrm{j}$ occur in specified Spatial relationship.

- The sum of all the elements $i, j$ of the GLCM will be the total number of times the specified spatial relationship occurs in $\mathrm{W}$.

- $\quad$ Calculate the selected Feature. This calculation uses only the values in the GLCM. For e.g.

- Energy,

- Entropy,

- Contrast, 


\section{IJARCCE}

- Homogeneity,

- Correlation

- $\quad$ The sample $\mathrm{s}$ in the resulting virtual variable is replaced by the value of this calculated feature.

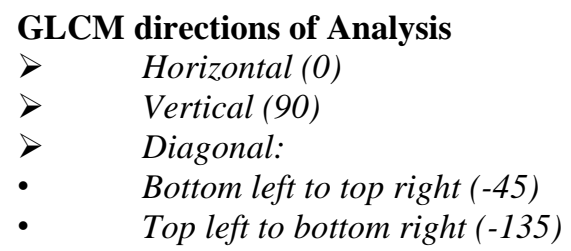

„, Denoted $P, P, P, \& P$ Respectively.

Ex. $P_{0}(i, j)$.

GLCM of an image is computed using a displacement vector d, defined by its radius $\delta$ and orientation $\theta$.

Consider a 4x4 image represented by figure 1a with four gray-tone values 0 through 3 . A generalized GLCM for that image is shown in figure $1 \mathrm{~b}$ where \# $(\mathrm{i}, \mathrm{j})$ stands for number of times $\mathrm{i}$ and $\mathrm{j}$ have been neighbors satisfying the condition stated by displacement vector $\mathrm{d}$.

\section{Energy}

- $\quad$ called Uniformity or Angular second moment.

- Measures the textural uniformity that is pixel pair repetitions.

- Detects disorders in textures.

- $\quad$ Energy reaches a maximum value equal to one.

\section{Entropy}

$$
\text { Energy }=\sum_{i} \sum_{j} p_{i j}^{2}
$$

- $\quad$ Measures the disorder or complexity of an image.

- The entropy is large when the image is not texturally uniform.

- Complex textures tend to have high entropy.

- $\quad$ Entropy is strongly, but inversely correlated to energy.

- $\quad$ Entropy $($ ent $)=-\sum_{i} \sum_{j} p_{i j} \log _{2} p_{i j}$

\section{Probabilistic Neural Network}

Performance of the PNN classifier was evaluated in terms of training performance and classification accuracies. This network is a kind of radial basis network and it gives fast and accurate classification and is a promising tool for classification of the defects from quality material. Existing weights will never be alternated but only new vectors are inserted into weight matrices when training. So it can be used in real-time. Since the training and running procedure can be implemented by matrix manipulation, the speed of PNN is very fast.

\section{Architecture of Probabilistic Neural Network}

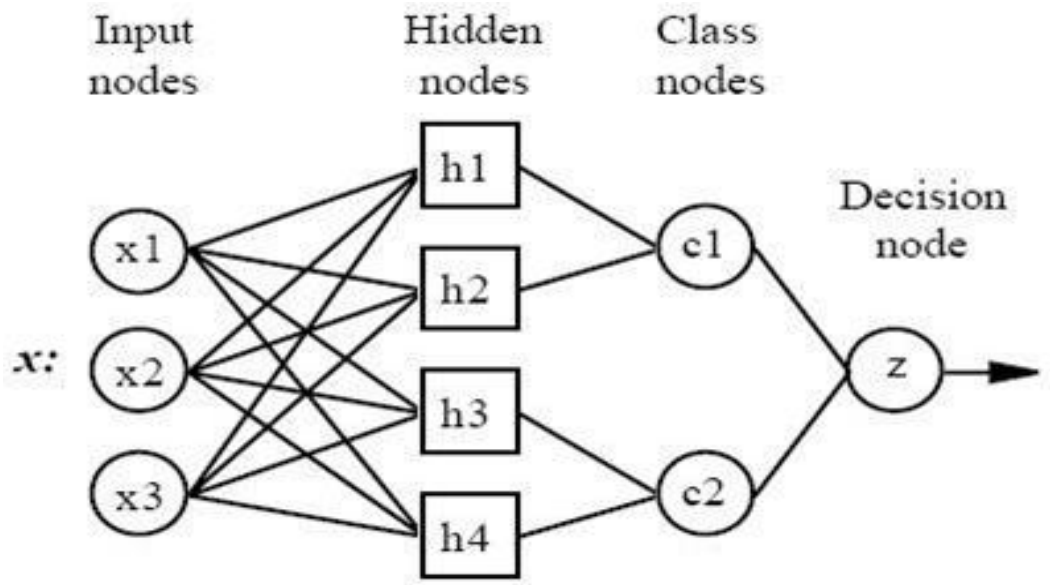

Figure 3 Probabilistic Neural Networks 
PNN is often used in classification problems. When an input is present, the first layer computes the distance from the input vector to the training input vectors. This produces a vector where its elements indicate how close the input is to the training input. The second layer sums the contribution for each class of inputs and produces its net output as a vector of probabilities. Finally, a compete transfer function on the output of the second layer picks the maximum of these probabilities, and produces a 1 (positive identification) for that class and a 0 (negative identification) for nontargeted classes.

\section{Input Layer}

Each neuron in the input layer represents a predictor variable. In categorical variables, $N-1$ neurons are used when there are' n' number of categories. It standardizes the range of the values by subtracting the median and dividing by the inter quartile range Then the input neurons feed the values to each of the neurons in the hidden layer.

\section{Pattern Layer}

This layer contains one neuron for each case in the training data set. It stores the values of the predictor variables for the case along with the target value. A hidden neuron computes the Euclidean distance of the test case from the neuron's center point and then applies the radial basis function kernel function using the sigma values.

\section{Summation Layer}

For PNN networks there is one pattern neuron for each category of the target variable. The actual target category of each training case is stored with each hidden neuron; the weighted value coming out of a hidden neuron is fed only to the pattern neuron that corresponds to the hidden neuron's category. The pattern neurons add the values for the class they represent.

\section{Output Layer}

The output layer compares the weighted votes for each target category accumulated in the pattern layer and uses the largest vote to predict the target category. PNN networks are relatively insensitive to outliers and they networks generate accurate predicted target probability scores. PNNs approach bayes optimal classification.

\section{IMPLEMENTATION AND RESULTS}

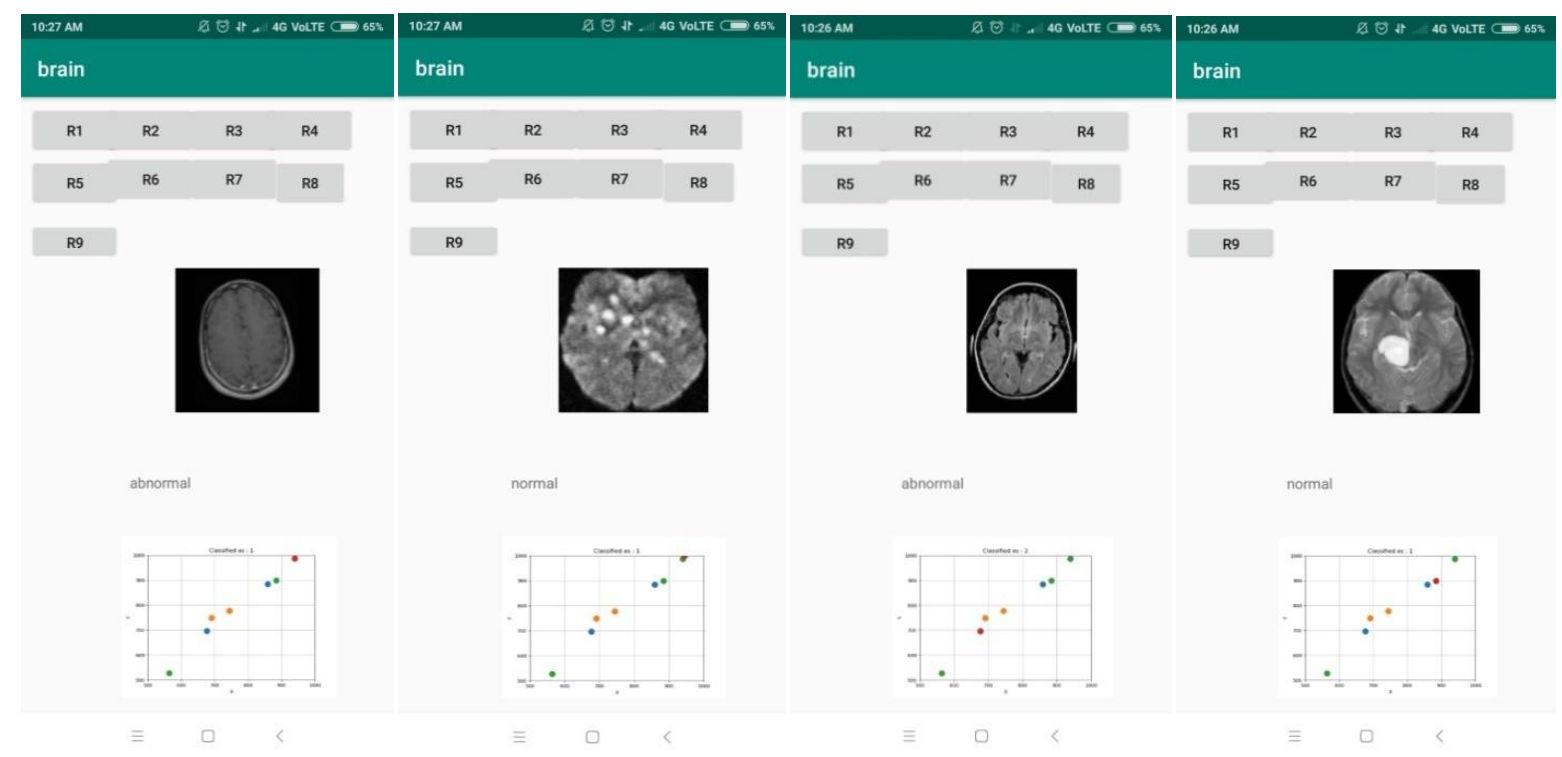

Figure 4.
a.
Abnormal brain
b. Normal Brain
c. Abnormal brain
d. Normal Brain

\section{CONCLUSION AND FUTURE WORK}

In MR images, the amount of data is too much for manual interpretation and analysis. During past few years, brain tumour segmentation in Magnetic Resonance Imaging (MRI) has become an emergent research area in the field of medical imaging system. The classification is performed on the tissues to three classes of normal, begin and malignant Accurate detection of size and locations of brain tumour plays a vital role in the diagnosis of tumour. The diagnosis 
Vol. 8, Issue 2, February 2019

method consists of four stages, Pre-processing of MR images, future extraction and classification. The features selection are based on discrete wavelength transformation method and future extraction is based GLCM. In the last stage, probabilistic neural network is employed to classify the normal and abnormal brain. After that normal image store on cloud and move towards on android application.

\section{REFERENCES}

[1]. Ali ARI*,Davut HANBAY, "Deep learning based brain tumour classification and detection system”,. (Turkish Journal of Electrical Engineering \& Computer Sciences 2019).

[2]. K.Kamnitas Et al., "Efficient multi scale 3D CNN with fully connected CRF for accurate brain lesson segmentation", Medical imaging analysis, Feb 2017.

[3]. A.Cameron, F.Khalvati and M.A.Haider, "Maps-A Quantitative radiomics approach for a prostate cancer detection", IEEE transactions on biomedical engineering, June 2016.

[4]. S.Pereia, A.pinto and C.A.silva,"Brain tumor segmentation using convolutional neural networks in MRI images", IEEE transactions on medical imaging, May 2015

[5]. Saif D. Salman \& Ahmed A. Bahrani, "Segmentation of tumour tissue in grey medical images using watershed transformation method", Biomedical Engineering Department, Al-Khwarizmi College of Engineering, Baghdad University, Baghdad, Iraq. (2016)

[6]. R.Preetha and G.R.Suresh Performance Analysis of Fuzzy C Means Algorithm in Automated Detection of Brain Tumor, World Congress on Computing and Communication Technologies,2014.

[7]. M.C. Jobin Christ and R.M.S. Parvathi ,"Segmentation of Medical Image using Clustering and Watershed Algorithms “, American Journal of Applied Sciences 8 (12): 1349-1352, 2011 ISSN 1546-9239 (C) 2011 Science Publications

[8]. Alan Jose, S.Ravi, M.Sambath,” Brain Tumor Segmentation Using K-Means Clustering And Fuzzy C-Means Algorithms And Its Area Calculation", International Journal of Innovative Research in Computer and Communication Engineering ,2011

[9]. Mrs.MamataS.Kalas, "An Artificial Neural Network for Detection of Biological Early Brain Cancer", International Journal of Computer Applications (2010) 